\title{
LA LIBERTAD CIVIL COMO CONDICIÓN DE POSIBILIDAD DE LA AUTONOMÍA MORAL. SOBRE LA APORTACIÓN DE FREUD AL PLANTEAMIENTO PRÁCTICO DE KANT ${ }^{1}$
}

\author{
Luis Alegre ZAHONERO*
}

\begin{abstract}
"Seguimos siendo psicoanalistas. Seguimos pensando que la verdad, solamente la verdad y nada más que la verdad cura de verdad"

Nestor Braunstein.
\end{abstract}

\begin{abstract}
RE SUM E N : Es frecuente buscar en Freud a un crítico de la llustración y, en particular, del modo de pensar la moral heredero del planteamiento kantiano. Sin embargo, Freud se limita a descubrir en la naturaleza humana un importante obstáculo del que el proyecto ilustrado no se podrá ya desentender. Este obstáculo, que afecta de un modo decisivo al modo como entender el paso a la mayoría de edad, remite a la existencia de un determinado reino de la heteronomía cuyo rasgo más característico es su capacidad para suplantar la voz de la autonomía moral: al igual que la ley moral en Kant, el superyó - una vez ha interiorizado en la infancia y asumido como propios determinados contenidos de cultura
\end{abstract}

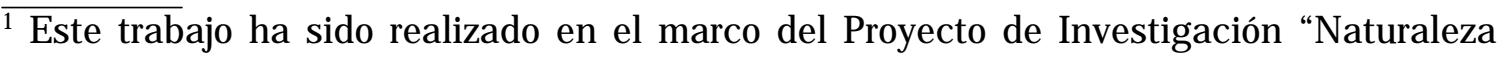
humana y comunidad. Una investigación, a partir de Kant, sobre los principios antropológicos del cosmopolitismo" (HUM 2006-04909), financiado por el MICINN.

* Doctor en Filosofía por la Universidad Complutense de Madrid, donde presentó la Tesis Ciudadanía y clase social en 'El capital' de K. Marx. J unto a Carlos Fernández Liria, ha escrito los libros Educación ético-cívica (2008), Educación para la Ciudadanía. Democracia, capitalismo y Estado de derecho (2007) y Comprender Venezuela, pensar la democracia. El colapso moral de los intelectuales occidentales (2006), obra que recibió el Premio Nacional del Libro de Venezuela y mención honorífica en el Premio Libertador al Pensamiento Crítico. Próximamente, publicarán un estudio sobre Marx titulado EI orden de 'El capital'.
} 
(independientemente de su racionalidad y de su posible validez como leyes universales)- también ordena poderosamente sin prometer nada (externo) ni amenazar con nada (externo). En estas condiciones, el proyecto de investir a la razón como autoridad suprema (proyecto que Freud y Kant comparten) depende de la existencia de una organización republicana que garantice un programa pedagógico para el que "mayoría de edad" no signifique llamar "yo" a la cristalización interior de las costumbres de los ancestros sino a la capacidad efectiva de guiarse por la propia razón (lo cual, tras el descubrimiento de los mecanismos elementales del psiquismo, se convierte en un imperativo no sólo moral sino también terapéutico).

PALA B RA S CLAVE : Freud. Kant. M ayoría de edad. M inoría de edad. Ilustración.

\section{Introducción}

"¿Qué hay en mí que hace que pueda sacrificar las más íntimas seducciones de mis impulsos y todo deseo que procede de mi naturaleza a una ley que no me promete como compensación ventaja alguna y con cuya infracción no amenaza ninguna pérdida, de suerte que, cuanto más la venero íntimamente, más severo sea el mandato y menos ofrezca en cambio?" 2 . El descubrimiento por parte de Freud de los mecanismos elementales del psiquismo introduce un importante imprevisto en el planteamiento de Kant: esa voz que habla a la voluntad de una forma tan clara, tan difícil de ahogar y tan perceptible hasta para los hombres más comunes ${ }^{3}$, esa voz que hace temblar incluso al criminal más audaz ${ }^{4}$, unas veces es la voz de la razón pero, otras, la voz de la autoridad paterna (asumida sin más como propia durante la infancia). Este descubrimiento, sin duda, interpela a Kant de un modo muy directo: esa voz del deber, que habla con tanta nitidez y solemnidad, no siempre merece llenar el alma de creciente admiración y respeto ${ }^{5}$. No siempre es tan fácil distinguir la voz de la razón (anunciadora para Kant de una procedencia divina ${ }^{6}$ ) de la voz de la religión (portadora para Freud de la neurosis obsesiva de la colectividad humana) ${ }^{7}$.

En el presente artículo trataremos de demostrar que Freud no niega la distinción entre una y otra (es decir, entre los imperativos inapelables de la

\footnotetext{
${ }^{2}$ Von einem neuerdings erhobenen vornehmen Ton in der Philosophie. AA: VII, 402.

${ }^{3} \mathrm{KpV}, \mathrm{AA}: \mathrm{V}, 35$.

${ }^{4} \mathrm{KpV}, \mathrm{AA}: \mathrm{V}, 80$.

${ }^{5} \mathrm{KpV}, \mathrm{AA}: \mathrm{V}, 161$.

${ }^{6}$ Die Religion innerhalb der Grenzen der bloßen Vernunft , AA: VI, 49.

${ }^{7}$ Die Zukunft einer Illusion, Gesammelte Werke, XIV, p. 367 [EI porvenir de una ilusión, Obras Completas, XXI, p. 43]
} 
razón y las prescripciones más o menos arbitrarias de la cultura) ${ }^{8}$. Es frecuente (aunque desconcertante) que se identifique sin más el superyó en Freud con los imperativos prácticos que analizada por Kant. Sin embargo, como trataremos de defender, en el planteamiento de Freud cabe distinguir con rotundidad entre exigencias de la razón y prescripciones de la cultura como instancias diferentes que pugnan por ocupar el lugar de la conciencia moral. Freud descubre que los mandatos de la religión, la cultura y la autoridad paterna suplantan con facilidad, en la edad adulta, a los mandatos de la razón y la libertad. Dado el peculiar modo que tenemos los humanos de insertarnos en el lenguaje, se trata de una impostura difícil de evitar. La infancia y la peculiar constitución del psiquismo no ponen fáciles las cosas al proyecto de servirse del propio entendimiento sin la guía de otro ${ }^{9}$. Freud, sin duda, es un firme defensor de este programa político de la llustración, pero pone sobre la mesa dificultades que van mucho más allá de afirmar que "la pereza y la cobardía son las causas de que una gran parte de los hombres permanezca, gustosamente, en minoría de edad a lo largo de la vida, a pesar de que hace ya tiempo la naturaleza los liberó de dirección ajena (naturaliter majorennes); y por eso es tan fácil para otros erigirse en sus tutores. ¡Es tan cómodo ser menor de edad!"10. Frente a este reproche kantiano, el psicoanálisis defiende más bien que, en contra de lo que pudiera parecer, en absoluto es "tan cómodo" ser menor de edad. Por el contrario, la peculiar minoría de edad que acompaña a la religión genera un notable malestar. Pero, eso sí, por intenso que sea el malestar que produce esta minoría de edad de la que, digamos, "el psiquismo" es culpable, no siempre bastan el valor y la audacia para desactivar los mecanismos por los que se impone. En este sentido, el proyecto ilustrado se encuentra con importantes obstáculos que dificultan el "tranquilo y duradero gobierno de la razón sobre el entendimiento y los sentidos"11 propuesto por Kant. Pero la presencia de esos obstáculos, por difíciles de vencer que puedan resultar, no impide que Freud siga considerando, ciertamente, que "nuestra mejor esperanza para el futuro es que el intelecto -el espíritu científico, la razón- establezca con el tiempo la dictadura (Diktatur) dentro de la vida anímica"12.

\footnotetext{
${ }^{8}$ En efecto, como sostiene Ramón Sanz Ferramola, "en general se da por supuesto el vínculo, sin mayores cuestionamientos, entre 'imperativo categórico' de Kant y 'superyó' o 'complejo de Edipo' o 'moral del psicoanálisis'." "El imperativo categórico de Kant en Freud", Fundamentos en humanidades, n. 3, p. 168, 2001.

${ }^{9}$ Beantwortung der Frage: Was ist Aufklärung?, AA: VIII, 35.

${ }^{10}$ Id.

${ }^{11} \mathrm{KrV}$ A 465/B493.

${ }^{12}$ Neue Folge der Vorlesungen zur Einführung in die Psychoanalyse, Gesammelte Werke, XV, p. 185 [Nuevas lecciones introductorias al psicoanálisis, Obras Completas, XXII, p. 158.]
} 
En el presente artículo, trataremos de mostrar, por un lado, la plena coincidencia entre los proyectos prácticos de Kant y Freud ${ }^{13}$. Por otro lado, trataremos de señalar una aportación fundamental de Freud al mismo: el descubrimiento de los mecanismos elementales del psiquismo pone de manifiesto que la libertad civil (y las instituciones educativas que le corresponden) no es sólo condición del Estado de derecho sino, incluso, de la propia autonomía moral (en un sentido kantiano) ${ }^{14}$.

\section{Cultura y restricción}

En el planteamiento de Freud, el objetivo de la cultura es, por un lado, proteger al hombre de las fuerzas de la naturaleza y, por otro, regular los vínculos recíprocos entre los hombres ${ }^{15}$. No cabe duda de que, desde cierto punto de vista, no dejamos en ningún momento de ser "naturaleza": debemos protegernos del frío y la intemperie, nos reproducimos (individualmente) mediante la ingesta de alimentos y nos reproducimos (como especie) mediante el sexo. Pero, evidentemente, no somos sólo naturaleza: el hombre no se protege "naturalmente" de la naturaleza ni regula "naturalmente" sus relaciones con los otros. En efecto, el hombre no domina las fuerzas de la naturaleza, por ejemplo, generando pelo ni fortaleciendo la mandíbula, sino produciendo herramientas, desarrollando la técnica y generando una intensa división del trabajo. Del mismo modo, las relaciones de los hombres entre sí están mucho más mediadas por las instituciones que, por ejemplo, por las feromonas.

En este sentido, ningún individuo puede por menos que reconocer el general interés humano de la civilización: el hombre necesita la cultura

\footnotetext{
${ }^{13}$ Bien es verdad que, como señala Zeljko Loparic, hay una diferencia fundamental entre ambos: en el proyecto de Freud no hay ninguna "razón de principio para excluir la posibilidad de que, en el futuro, la ciencia de la conciencia, tanto teórica como moral, pueda ser formulada en términos meramente físicos, posibilidad explícitamente excluida por Kant". Zeljko Loparic, "De Kant a Freud: um roteiro", en Naturaleza humana, 5, 2003, p. 245. Sin embargo, si atendemos no tanto a lo que Freud cree que podría llegar a hacerse en un futuro sino a la que efectivamente hace, las diferencias entre el planteamiento de ambos se reduce notablemente.

${ }^{14}$ El presente artículo concierne principalmente al terreno de la razón práctica. Para un análisis del modo como la metapsicología freudiana encuentra también su fundamento especulativo en el planteamiento de Kant, cfr. Leopoldo Fulgencio, "Kant es as especulações metapsicológicas em Freud". Kant e-Prints, v. 2, n. 9, p. 1-31, 2003.

${ }^{15}$ Das Unbehagen in der Kultur, Gesammelte Werke, XIV, p. 448-449 [EI malestar en la cultura, Obras Completas, XXI, p. 88]; Die Zukunft einer Illusion, Gesammelte Werke, XIV, p. 326 [EI porvenir de una ilusión, Obras Completas, XXI, p. 6].
} 
porque necesita la vida en común. A hora bien, al mismo tiempo, el hombre es virtualmente un enemigo de la civilización: la vida en común impone necesariamente restricciones a la libre satisfacción de todos los posibles deseos y, en esa medida, implica sacrificios y renuncias. Ciertamente, la propia cultura tiene un notable margen de acción en la determinación de los propios deseos y en la articulación de los mecanismos con los que satisfacerlos. El hombre no puede ni siquiera desear al margen de la cultura, pero la cultura tampoco tiene una capacidad de acción ilimitada en la conformación y canalización de los deseos. En este sentido, ninguna cultura se podrá desentender jamás de la necesidad de sus miembros de obtener satisfacción sexual y de disponer de los bienes necesarios con los que garantizar la propia subsistencia. Así pues, la cultura tiene un amplio margen para conformar el deseo sexual y para regular la tenencia de bienes, pero no un poder absoluto sobre estos deseos: puede configurar y canalizar el deseo sexual de modos diversos, algunos de ellos extraordinariamente restrictivos, pero no podrá en ningún caso suprimirlo; puede organizar la tenencia de bienes de muchas formas, algunas muy opresivas para parte de sus miembros, pero hay límites mínimos que la naturaleza impone para la reproducción del organismo. En este sentido, por mucho que se asigne a la cultura un poder constituyente sobre los propios deseos, hay siempre un reducto de naturaleza que no dejará jamás de hacer valer sus aspiraciones. La cultura, sin duda, conforma y constituye, pero no cualquier horma es apta para albergar a la naturaleza humana.

Así pues, si no quiere quebrar la naturaleza humana o resultar incompatible con ella, la cultura ha de abrir siempre un espacio a los impulsos afectivos del hombre y lo que por ellos es determinado. Pero, al mismo tiempo, no puede por menos de introducir restricciones que implican la renuncia de cada uno a la satisfacción ilimitada de esos impulsos. La civilización impone, pues, renuncias necesarias para hacer posible la vida en común. Y, ciertamente, estas renuncias implican un sacrificio. En efecto, sólo cabría negar ese elemento de sacrificio si se defendiese una comunidad tan absolutamente hermética y cerrada sobre sí misma que no cupiese ya siquiera la posibilidad de desear algo distinto de lo prescrito por la propia comunidad (o, en definitiva, si se negase el carácter irreductible de cierto elemento de naturaleza en la constitución humana) ${ }^{16}$.

\footnotetext{
${ }^{16}$ En esta dirección, el intento de establecer una continuidad material entre virtud y felicidad (a través de la comunidad y la familia) defendida por J uan Bautista Fuentes sería el non plus ultra del pensamiento reaccionario (al menos hasta donde el trágico siglo XX permitió dar de sí a los teóricos del nazismo, del nacional-catolicismo y, a su modo, del maoismo; cabe en cualquier caso esperar que el siglo XXI no amplíe ese margen). Cfr. J uan Bautista Fuentes, "De Kant a Freud: Ia formación del sujeto modernista en el seno de las crisis románticas del pensamiento kantiano", revista Pensamiento (en prensa).
} 
Con el objetivo de ilustrar el sentido y la justificación de este sacrificio, Freud propone el siguiente relato:

I maginemos canceladas sus prohibiciones: será lícito escoger como objeto sexual a la mujer que a uno le guste, eliminar sin reparos a los rivales que la disputen o a quienquiera que se interponga en el camino; se podrá arrebatarle a otro un bien cualquiera sin pedirle permiso: iqué hermosa sucesión de satisfacciones sería entonces la vida! Claro que enseguida se tropieza con la inmediata dificultad: los demás tienen justamente los mismos deseos que yo, y no me dispensarán un trato más considerado que yo a ellos. Por eso, en el fondo, sólo un individuo podrá devenir ilimitadamente dichoso mediante esa cancelación de las limitaciones culturales: un tirano, un dictador, que haya atraído hacia sí todos los medios de poder; y ese individuo, además, tendrá todas las razones para desear que los otros obedezcan al menos a este solo mandamiento cultural: «N o matarás». Pero, icuán impensable, cuán miope en todo caso aspirar a una cancelación de la cultura! Sólo quedaría el estado de naturaleza, que es mucho más difícil de soportar. Es verdad que la naturaleza no nos exigía limitar en nada nuestras pulsiones, las consentía; pero tiene su modo, particularmente eficaz, de limitarnos: nos mata, a nuestro parecer de una manera fría, cruel y despiadada, y acaso a raíz de las mismas ocasiones de nuestra satisfacción. justamente por esos peligros con que la naturaleza nos amenaza nos hemos aliado y creado la cultura, que, entre otras cosas, también debe posibilitarnos la convivencia. Y por cierto la principal tarea de la cultura, su genuina razón de existir, es protegernos de la naturaleza. ${ }^{17}$

Cualquier narración de este tipo, inevitablemente, incorpora algún elemento mítico. Cabría argumentar que es sencillamente falso que, en ausencia de restricciones, todos quisiéramos violar, robar y matar a nuestros vecinos. No obstante, lo que sí parece más o menos evidente es que "la ley sólo prohíbe a los seres humanos aquello que podrían llevar a cabo bajo el esforzar de sus pulsiones. No hace falta que sea prohibido y castigado por la ley lo que la naturaleza misma prohíbe y castiga. Por eso podemos suponer tranquilamente que unos delitos prohibidos por una ley son tales que muchos hombres los cometerían Ilevados por sus inclinaciones naturales". ${ }^{18}$ Para la consistencia del planteamiento de Freud no es necesario sostener que los hombres deseen (de hecho) violar todas las restricciones que impone la cultura. Por el contrario, al argumento de Freud le basta demostrar que no es

\footnotetext{
${ }^{17}$ Die Zukunft einer Illusion, Gesammelte Werke, XIV, p. 335-336 [EI porvenir de una ilusión, Obras Completas, XXI, p. 15].

${ }^{18}$ Totem und Tabu, Gesammelte Werke, IX, p. 150 [Totem y Tabu, Obras Completas, XIII, p. 126].
} 
imposible que los individuos puedan albergar deseos contrarios a los principios elementales de la civilización.

En cualquier caso, las renuncias que impone la civilización implican sacrificios pero, sin duda, preferibles a la ausencia completa de normas. La libertad irrestricta del estado de naturaleza implica, en realidad, la ausencia absoluta de libertad (excepto, en todo caso, para el más fuerte, y sólo de un modo provisional). La ausencia de regulación desemboca necesariamente en la situación más restrictiva que cabe imaginar. Nadie puede querer que la libertad se ejerza con carácter general sin más límites que los naturales. En efecto, cada uno podrá desear quedar fuera de esa restricción y convertir su propia vida en una "hermosa sucesión de satisfacciones". Pero si "los demás tienen justamente los mismos deseos que yo", la exención general haría inviable la vida en común. La cultura impone necesariamente restricciones y sacrificios a la libre satisfacción de los individuos pero, ciertamente, suprimida la civilización, "sólo quedaría el estado de naturaleza, que es mucho más difícil de soportar". En ese sentido, nos dice Freud que "el primer requisito cultural", el requisito mínimo de la civilización es "la justicia, o sea, la seguridad de que el orden jurídico ya establecido no se quebrantará para favorecer a un individuo"19

\section{Razón y religión: dos posibles órdenes de sentido}

Así pues, no se puede por menos que desear cierta limitación a la libertad individual. A hora bien ¿según qué reglas cabe establecer esta restricción?

Si las normas se basasen exclusivamente en exigencias de la razón y del lenguaje (algo que, ciertamente, está lejos de ser el caso), las restricciones responderían a un principio simple: basta comprender la lógica de los pronombres personales para estar forzado a admitir que cualquier "yo" (por

\footnotetext{
${ }^{19}$ Das Unbehagen in der Kultur, Gesammelte Werke, XIV, p. 455 [EI malestar en la cultura, Obras Completas, XXI, p. 94]. Ciertamente, Freud tiene todo el cuidado en señalar que, en principio, esto no implica un pronunciamiento sobre el valor ético de semejante derecho ya que, con frecuencia, ese sistema de reglas viene a cristalizar en realidad el dominio de un grupo de poder sobre el resto en el interior de una comunidad. Sin embargo, "desde este punto, el desarrollo cultural parece dirigirse a procurar que ese derecho deje de ser expresión de la voluntad de una comunidad restringida - casta, estrato de la población, etnia - que respecto a otras masas, acaso más vastas, volviera a comportarse como lo haría un individuo violento. El resultado último debe ser un derecho al que todos -al menos todos los capaces de vida comunitaria - hayan contribuido con el sacrificio de sus pulsiones y en el cual nadie -con la excepción ya mencionada - pueda resultar víctima de la violencia bruta" (Id.).
} 
muy especial que sea para sí mismo) no es, para cualquier otro, más que un "tú" que, a su vez, es un "yo" cualquiera, lo mismo que "él". Puede que cada uno, ciertamente, tenga el máximo interés en darse un trato preferencial a sí mismo pero, desde el punto de vista de la escueta lógica que pone en juego cualquier lenguaje, resulta que cualquier "yo" (por mucho que sea, con diferencia, el "favorito de sí mismo") es, como "tú" o como "él", un "yo" cualquiera. Esta escueta lógica bastaría por sí sola para establecer un sistema mínimo de reglas infranqueables. En efecto, hace falta quebrar la consistencia misma del lenguaje para querer, por ejemplo, que ciertas restricciones sean impuestas a todos excepto a uno mismo (pues, en estricta lógica, sería tanto como reclamar una norma de validez para todos excepto para cada uno). Cada uno, en la medida en que sea el favorito de sí mismo, puede querer hacer excepciones a las reglas que querría ver en vigor para todos. Sin embargo, la alternativa "estrictamente racional" a la selva pasa por el mandato de acatar las reglas de validez general. La escueta lógica de los pronombres personales, ciertamente, no da ninguna información sobre el contenido de los deseos en cada caso. Pero sí es posible deducir a partir de ella ciertos principios mínimos ineludibles para limitar la libertad y los derechos de todos para hacerlos compatibles con los derechos y libertades de cada uno. Esto mismo es, en efecto, lo que sostiene Kant al afirmar que "la razón pura es por sí sola práctica"20.

Sin embargo, las comunidades humanas están lejos de regirse por preceptos netamente racionales. Los humanos, que nacemos sin saber hablar, no accedemos a la razón más que a través de una lengua materna que, inevitablemente, además de los pronombres personales, lleva ya siempre en el mismo lote todas las costumbres de la tribu, los imperativos de la cultura y los mandatos de la religión. Entre los mandatos de las costumbres van siempre mezclados (de un modo indiferenciado para los propios hablantes) exigencias de la razón y otras prescripciones de todo tipo. Incorporarse a una cultura y a un lenguaje no implica sólo asumir ciertos preceptos racionales sino, al mismo tiempo, asimilar todas las ocurrencias ancestrales de la autoridad de los antepasados. Freud, ciertamente, intenta que no se confundan ambas cosas:

Para volver a la ética, diríamos a modo de conclusión: una parte de sus preceptos se justifican con arreglo a la razón por la necesidad de limitar los derechos de la comunidad frente a los individuos, los derechos de los individuos frente a la comunidad y los de los individuos entre sí. Sin embargo, lo que en la ética nos aparece grandioso, misterioso, cosa

${ }^{20} \mathrm{KpV}, \mathrm{AA}: \mathrm{V}, 31$. 
místicamente evidente, debe tales caracteres a su nexo con la religión, a su origen el la voluntad del padre. ${ }^{21}$

Para Freud resulta crucial distinguir entre los preceptos éticos, que se sostienen sobre un fundamento racional, y los preceptos religiosos, que carecen de él. En efecto, cualquier intento de mezclar o confundir ambas cosas podría tener consecuencias indeseables. Es fundamental para Freud no basar los imperativos racionales de la ética en fundamentos de orden religioso. Es peligroso hacer depender la validez de los mandatos éticos de un fundamento relativo a la autoridad del padre. El fundamento de la ética, pues, no puede en ningún caso ser Dios. Así, "los reclamos éticos que la religión pretende sancionar piden más bien otro fundamento, pues son indispensables para la sociedad humana y es peligroso atar su observancia a la fe religiosa"22. Ciertamente, si el cumplimiento de las reglas éticas se hace depender de aceptación de distintos catecismos y doctrinas y de la obediencia a cultos históricos, se corre el riesgo de erosionar (en paralelo a la probable erosión de la autoridad clerical) la potencia de los mandatos éticos que sí tienen un fundamento estrictamente racional. A este respecto, Freud considera evidente que "cabe exigir a todos los hombres que empleen las dotes de la razón que poseen" 23 , pero no cabe hacer lo mismo con la religión. Precisamente por eso, para Freud, "no hay instancia alguna superior a la razón" 24.

\footnotetext{
${ }^{21}$ Der Mann Moses und die monotheistische Religion, Gesammelte Werke, XVI, p. 230. [M oisés y la religión monoteísta, Obras Completas, XXIII, p. 118]. Según se avanza en la obra de madurez de Freud, va resultando más evidente su compromiso con esta distinción que, sin embargo, la tradición psicoanalista no siempre ha hecho. En este sentido, María J osé Callejo no sólo tiene razón sino que se coloca en la más estricta fidelidad al pensamiento de Freud cuando, en su artículo "Ley, deseo y libertad. Notas sobre Lacan y la Crítica de la razón práctica" (documento de trabajo del grupo Metafísica, Crítica y Política, dirigido por J uan Manuel Navarro Cordón) reclama que "el psicoanálisis habría de buscar la cuidadosa disociación del dispositivo del superyo y guardar el sitio vacío para los datos puros de la Razón pura práctica, ayudar a distinguir en la vida moral lo que no es sino pago del precio exigido por el Otro (Ello) por su renuncia y acomodación a la estructura del lenguaje, y las verdaderas exigencias de la Razón".

${ }^{22}$ Neue Folge der Vorlesungen zur Einführung in die Psychoanalyse, Gesammelte Werke, XV, p. 181[Nuevas lecciones introductorias al psicoanálisis, Obras Completas, XXII, p. 155].

${ }^{23}$ Die Zukunft einer Illusion, Gesammelte Werke, XIV, p. 350 [EI porvenir de una ilusión, Obras Completas, XXI, p. 28]

${ }^{24}$ Id. Debe advertirse que Kant señala exactamente el mismo problema en La religión dentro de los límites de la mera razón, cuya idea central es, ciertamente, que "la moral no necesita ni de la idea de otro ser por encima del hombre para conocer su deber ni de otro motivo impulsor que la ley misma para observarlo" (Die Religion innerhalb der Grenzen
} 
A hora bien, estos dos órdenes o, como dice Freud, "dos diversas motivaciones" (una de orden racional relativa a la igualdad de derechos entre los diferentes individuos y otra de carácter ancestral relativa a la autoridad del padre $)^{25}$, relativamente fáciles de distinguir desde un punto de vista analítico, se encuentran siempre, constitutivamente, mezcladas de un modo indiferenciado para el hablante de cualquier lengua materna. Quienes, como los humanos, adquirimos al mismo tiempo tanto la razón como la cultura (pues, en definitiva, eso es lo que significa aprender a hablar) no podemos distinguir con tanta facilidad en nuestra vida cotidiana los mandatos de ambas. Así pues, lo que ordena el superyó lleva incrustado, al mismo tiempo que todos los mandatos que derivan del hecho mismo de estar atravesados por el lenguaje (y, en esa medida, por la razón), una cantidad indeterminada de preceptos que carecen de toda base racional.

Este resultado es inevitable para esos seres racionales en los que el punto de partida no es ya el lenguaje sino una masa informe de exigencias instintiva que, en todo caso, tiene miedo al castigo y, eso sí, tiene la capacidad de aprender a hablar. En estas condiciones, la infancia, digamos, física o natural (esa de la que nadie es culpable) implica necesariamente un periodo de restricciones impuestas desde fuera. Ciertamente el poder de la autoridad externa en la infancia para determinar lo permitido y lo prohibido es (y no puede ser de otro modo) casi absoluto. ''M alo' es, en un comienzo, aquello por lo cual uno es amenazado con la pérdida del amor"26.

En el niño pequeño la situación nunca puede ser otra; pero es también la de muchos adultos, apenas modificada por el hecho de que la comunidad humana global remplaza en ellos al padre 0 a ambos progenitores. Por eso

der bloßen Vernunft , AA: VI, 3), es decir, que la moral "no necesita en modo alguno de la Religión" (id.). Por el contrario, es la verdadera religión la que debe basarse (al igual que la moral) exclusivamente en principios de la razón. En efecto, Kant defiende que "la verdadera Religión única no contiene nada más que leyes, esto es: principios prácticos de cuya necesidad incondicionada podemos ser conscientes y que, por lo tanto, reconocemos como revelados por la Razón pura (no empíricamente). (...) A hora bien, tener esta fe estatutaria (que está en todo caso limitada a un pueblo y no puede contener la universal Religión del mundo) por esencial para el servicio de Dios y hacer de ella la condición suprema de la complacencia divina en el hombre es una ilusión religiosa, cuyo seguimiento es un falso servicio" (Ibid, 167-168).

${ }^{25}$ Der Mann Moses und die monotheistische Religion, Gesammelte Werke, XVI, p. 227 [M oisés y la religión monoteísta, Obras Completas, XXIII, p. 115].

${ }^{26}$ Das Unbehagen in der Kultur, Gesammelte Werke, XIV, p. 484 [EI malestar en la cultura, Obras Completas, XXI, p. 120]. 
se permiten habitualmente ejecutar lo malo que les promete cosas agradables cuando están seguros de que la autoridad no se enterará o no podrá hacerles nada, y su angustia se dirige sólo a la posibilidad de ser descubiertos. ${ }^{27}$

Hasta aquí, la cuestión no presenta mayor dificultad. A partir de este planteamiento, cabría denominar "menor de edad" (en el sentido político y moral acuñado por la llustración) a aquel que es gobernado de un modo exclusivo a través de la autoridad que impone la coacción externa, es decir, aquel que carece de autonomía para determinar por sí mismo las pautas morales a las que se autoexige obedecer. Y, ciertamente, habría sin duda que distinguir entre esa minoría de edad de la que nadie es culpable (y que corresponde, en efecto, a la etapa infantil que impone la naturaleza) y esa otra minoría de edad que se mantiene en la edad adulta. Por mucho que se haya alcanzado la madurez física cabría seguir llamando "menores de edad" a quienes se orientan exclusivamente por la autoridad externa (ya sea representada por el padre o por la comunidad en su conjunto). Esta primacía de la autoridad exterior (esta, digamos, "incapacidad de servirse del propio entendimiento sin la guía de otro") se pondría ante todo de manifiesto en la limitación de las restricciones al "temor de ser descubiertos".

A hora bien, para cualquier cultura, el paso a la edad adulta consiste en asumir como si fuesen propios e interiores los mandatos, por decirlo así, del padre (o la comunidad) que a cada uno le haya tocado en suerte (por irracionales y arbitrarios que fueran). Este hecho, ciertamente, interpela de un modo muy directo al proyecto político de la Ilustración, pone en cuestión a qué cabe llamar propiamente "mayoría de edad" y obliga a preguntarse qué margen le queda al hombre para "servirse de su propio entendimiento sin la guía de otro".

\section{El superyó religioso como autoridad despótica}

Sólo sobreviene un cambio importante cuando la autoridad es interiorizada por la instauración de un superyó. Con ello los fenómenos de la conciencia moral son elevados a un nuevo grado; en el fondo, únicamente entonces corresponde hablar de conciencia moral y sentimiento de culpa. En ese momento desaparece la angustia frente a la posibilidad de ser descubierto, y también, por completo, el distingo entre hacer el mal y quererlo; en efecto, ante el superyó nada puede ocultarse, ni siquiera los pensamientos. ${ }^{28}$

\footnotetext{
${ }^{27}$ Das Unbehagen in der Kultur, Gesammelte Werke, XIV, p. 484 [EI malestar en la cultura, Obras Completas, XXI, p. 121].

${ }^{28}$ Das Unbehagen in der Kultur, Gesammelte Werke, XIV, p. 484 [EI malestar en la cultura, Obras Completas, XXI, p. 121].
} 
Cabría pensar que el superyó, como nueva autoridad, no puede tener tendencia alguna a martirizar al yo. En definitiva, se trata de una autoridad interior íntimamente articulada con él. Sin embargo, el hecho de ser interior introduce pocas modificaciones respecto al modo como se ejerce la autoridad a través del miedo al castigo divino o el miedo a ser descubierto. A este respecto, la principal novedad no es una mayor indulgencia sino, simplemente, una mayor probabilidad de ser descubierto. "El superyó es sucesor y subrogador de los progenitores (y educadores) que vigilaron las acciones del individuo en su primer periodo de vida; continúa las funciones de ellos casi sin alteración"29. En este sentido, torturará al pecaminoso yo con el máximo rigor o será más indulgente con él en función del sistema de reglas del que el propio superyó no es más que una cierta cristalización. Y, ciertamente, la Religión (en la medida en que suele operar fura de los límites de la mera razón) no tiene por qué ser muy permisiva. De hecho, en todo tipo de catecismos y doctrinas clericales encontramos prohibiciones y preceptos abusivos, incapaces de encontrar un fundamento racional y que se desentienden por completo de la felicidad (e incluso de la salud; por no hablar de la libertad) de los sujetos. Lo que encontramos son regulaciones minuciosas de toda la organización de los deseos y, sobre todo, un sistema de castigos que no ha sido ni remotamente "civilizado" por el más elemental principio de proporcionalidad de la pena. Un sistema de castigos que, por ejemplo, puede condenar al fuego eterno por la práctica del onanismo. El problema es que, ciertamente, una vez desenvuelto en esa atmósfera, el superyó opera con la misma severidad. En este sentido, no es extraño que Freud sostenga que "en la tarea terapeútica nos vemos precisados muy a menudo a combatir al superyó y a rebajar sus exigencias" ${ }^{30}$. El funcionamiento de las pesadillas es, a este respecto, bastante ilustrativo. "El sueño de angustia es muchas veces un cumplimiento no disfrazado de deseo, no desde luego el de un deseo admisible, sino el de uno reprobado"31. Una vez incorporadas como propias las abusivas prescripciones de la religión, ya no hay tregua para el sujeto: satisfacer sus deseos incluso en sueños le es con frecuencia negado, pues el superyó los rechaza y los censura hasta el punto de lograr que la representación onírica, en vez de procurar placer, imponga un castigo. Desde el punto de vista de la conciencia y la razón las cosas nunca son tan restrictivas. Hay cierta sensatez común que se expresa

\footnotetext{
${ }^{29}$ Der Mann Moses und die monotheistische Religion, Gesammelte Werke, XVI, p. 224. [M oisés y la religión monoteísta, Obras Completas, XXIII, p. 113].

${ }^{30}$ Das Unbehagen in der Kultur, Gesammelte Werke, XIV, p. 503 [EI malestar en la cultura, Obras Completas, XXI, p. 138].

${ }^{31}$ Vorlesungen zur Einführung in die Psychoanalyse, Gesammelte Werke, XI, p. 222. [Introducción al psicoanálisis Obras Completas, XV, p. 198].
} 
con frecuencia, nos dice Freud, en la "razonable observación" de "es sólo un sueño"32. Pero el superyó (individual o cultural) no siempre atiende a razones. La severidad del superyó incorpora siempre un elemento irracional no sólo por el contenido de sus prescripciones (con frecuencia abusivas y arbitrarias) sino por castigar de una forma en la que, para obtener la absolución, no basta ni siquiera la renuncia a la satisfacción de los instintos: en la medida en que persista el mero deseo, se mantiene el castigo ${ }^{33}$.

"Con la severidad de sus mandamientos y prohibiciones se despreocupa demasiado de la felicidad del individuo" ${ }^{34}$. El superyó cultural y la religión no sólo ignora las exigencias de la libertad y las condiciones para la mayoría de edad ilustrada sino que

tampoco se cuida lo bastante de los hechos de la constitución anímica de los seres humanos, proclama un mandamiento y no preocupa si podrán obedecerlo. Antes bien, supone que al yo del ser humano le es psicológicamente posible todo lo que se le ordene, pues tendría un gobierno irrestricto sobre su ello. Ese es un error, y ni siquiera en los hombres llamados normales el gobierno sobre el ello puede llevarse más allá de ciertos límites. Si se exige más, se produce en el individuo rebelión o neurosis, o se lo hace infeliz. [... ] La cultura descuida todo eso; sólo amonesta: mientras más difícil la obediencia al precepto, más meritorio es obedecerlo. ${ }^{35}$

\section{El superyó racional como garantía de libertad}

¿Cabe seguir sosteniendo entonces el inapelable "si debo, sé que puedo"36 establecido por Kant? Para comprender precisamente la esencia del programa común a Kant y a Freud no se debe perder de vista que este lema kantiano se refiere exclusivamente, como es obvio, a esos mandatos que emanan de la pura razón y esto, en efecto, introduce diferencias fundamentales aceptadas por Freud: Ios mandatos de la razón no van en

\footnotetext{
${ }^{32}$ Vorlesungen zur Einführung in die Psychoanalyse, Gesammelte Werke, XI, p. 224 [Introducción al psicoanálisis Obras Completas, XV, p. 200].

${ }^{33}$ Das Unbehagen in der Kultur, Gesammelte Werke, XIV, p. 487. [EI malestar en la cultura, Obras Completas, XXI, p. 123].

${ }^{34}$ Das Unbehagen in der Kultur, Gesammelte Werke, XIV, p. 503 [EI malestar en la cultura, Obras Completas, XXI, p. 138].

${ }^{35}$ Das Unbehagen in der Kultur, Gesammelte Werke, XIV, p. 503 [EI malestar en la cultura, Obras Completas, XXI, p. 138].

${ }^{36}$ "Él juzga, pues, que puede hacer algo porque tiene conciencia de que debe hacerlo, y reconoce en sí mismo la libertad, que, sin la ley moral, hubiese permanecido desconocida para él", KpV, AA: V, 30.
} 
ningún caso a exceder los límites de lo que cualquier sujeto (hasta el más común) debe y puede reprimir.

En primer lugar, se debe señalar que la razón, aunque ordene con una voz sublime y que hace temblar, en rigor, prohíbe muy poca cosa. De hecho, la razón no puede en ningún caso prohibir más de lo estrictamente imprescindible para la vida en común. En este sentido, por ejemplo, no es práctica propia de la razón torturar a los menores con prescripciones asfixiantes sobre cada detalle de su vida sexual. Cuando Freud, como vimos, reclamaba "la dictadura de la razón sobre la vida anímica", Io hacía precisamente porque "la esencia de la razón garantiza que en tal caso no dejaría de asignar su lugar debido a las mociones afectivas de los seres humanos y a lo comandado por ellas" ${ }^{37}$.

Hasta tal punto considera Freud, digamos, "permisiva" a la razón que, cuando tiene que distinguir, entre los mandatos de la cultura, los que responden a un fundamento estrictamente racional de los que derivan de un sentido religioso (heredero social del espíritu y la autoridad del padre), Freud coloca incluso la prohibición del incesto fuera de los "preceptos que se justifican racionalmente". En efecto, cuando Freud distingue dos distintos órdenes de motivaciones, uno que deriva directamente de los mandatos del padre y otro que responde a las necesidades del pacto social, coloca dentro del segundo orden sólo los mandatos relativos a la igualdad de derechos. Todo lo demás (hasta la prohibición del incesto) queda dentro del primer orden de motivaciones ${ }^{38}$. Como vimos, sólo algunos preceptos "se justifican con arreglo a la razón por la necesidad de limitar los derechos de la comunidad frente a los individuos, los derechos de los individuos frente a la comunidad y los de los individuos entre sí"39. Fuera de ese escueto criterio, las prohibiciones sagradas tienen "un intensísimo tinte afectivo, pero ello, en verdad, sin un fundamento ajustado a la razón. En efecto, ¿por qué sería un crimen muy grave cometer incesto con una hija o una hermana, por qué sería este comercio sexual muchísimo más maligno que cualquier otro?"40

\footnotetext{
${ }^{37}$ Neue Folge der Vorlesungen zur Einführung in die Psychoanalyse, Gesammelte Werke, XV, p. 185. [Nuevas lecciones introductorias al psicoanálsis, Obras Completas, XXII, p. 158].

${ }^{38}$ Der Mann Moses und die monotheistische Religion, Gesammelte Werke, XVI, p. 227 [Moisés y la religión monoteísta, Obras Completas, XXIII, p. 115].

${ }^{39}$ Der Mann Moses und die monotheistische Religion, Gesammelte Werke, XVI, p. 230 [Moisés y la religión monoteísta, Obras Completas, XXIII, p. 118].

${ }^{40}$ Der Mann Moses und die monotheistische Religion, Gesammelte Werke, XVI, p. 228 [Moisés y la religión monoteísta, Obras Completas, XXIII, p. 116].
} 
Para Freud, pues, la razón no prohíbe más que los atentados contra la libertad y la integridad ajenas. Todo lo demás está permitido (o bien prohibido por la religión con un dudoso fundamento racional) y esto, ciertamente, tiene consecuencias inmediatas en lo relativo a la búsqueda y realización de la propia felicidad:

La felicidad, considerada en su sentido limitado, cuya realización parece posible, es meramente un problema de la economía libidinal de cada individuo. Ninguna regla al respecto vale para todos; cada uno debe buscar por sí mismo la manera en que pueda ser feliz. ${ }^{41}$

Esta formulación, ciertamente, coincide en su literalidad con la fórmula del principio de libertad (como primer principio a priori del estado civil) en Kant:

Nadie me puede obligar a ser feliz a su modo (tal como él se imagina el bienestar de otros hombres), sino que es lícito a cada uno buscar su felicidad por el camino que mejor le parezca, siempre y cuando no cause perjuicio a la libertad de los demás para pretender un fin semejante. ${ }^{42}$

Kant opone este principio de libertad (por el que se proscribe que alguien pueda dictar el modo como deben buscar la felicidad los demás) al de la autoridad que se ejerce al modo de un mandato paterno:

Un gobierno que se constituyera sobre el principio de la benevolencia para con el pueblo, al modo de un padre para con sus hijos, esto es, un gobierno paternalista (imperium paternale), en el que los súbditos -como niños menores de edad, incapaces de distinguir lo que es verdaderamente beneficioso o perjudicial - se ven obligados a comportarse de manera meramente pasiva, aguardando sin más del juicio del jefe de Estado cómo deban ser felices y esperando simplemente de su bondad que este también quiera que lo sean, un gobierno así es el mayor despotismo imaginable. ${ }^{43}$

Este reproche que Kant lanza contra el gobierno paternalista es exactamente el mismo reproche que Freud lanza contra la religión (en la que, claro está, Dios ejerce la autoridad como un padre): "Ia religión perjudica

\footnotetext{
${ }^{41}$ Das Unbehagen in der Kultur, Gesammelte Werke, XIV, p. 442. [EI malestar en la cultura, Obras Completas, XXI, p. 83 (sn)].

${ }^{42}$ Über den Gemeinspruch: Das mag in der Theorie richtig sein, taugt aber nicht für die Praxis, AA: VIII, 290.

${ }^{43}$ Über den Gemeinspruch: Das mag in der Theorie richtig sein, taugt aber nicht für die Praxis, AA: VIII, 290-291.
} 
este juego de elección y adaptación al imponer a todos por igual su camino único para conseguir la felicidad y protegerse del sufrimiento"44.

A este respecto, la principal diferencia entre Kant y Freud es el mayor detalle con el que éste analiza los mecanismos psíquicos por los que esa autoridad del padre (o de Dios) cristaliza en el superyó pudiendo condenar al hombre a una eterna minoría de edad de la que, en cierto modo, el psiquismo (y no tanto el sujeto) sería culpable.

En cualquier caso, tampoco es casualidad que el propio Kant sospechase que "la minoría de edad en cuestiones religiosas es, entre todas, la más perjudicial y humillante". ${ }^{45}$

En segundo lugar, la principal virtud de la razón no es sólo su recato y contención a la hora de imponer restricciones sino también lo, digamos, infinitamente "permisiva" que es a la hora de aceptar cualquier tipo de satisfacción sustitutoria que no produzca más que efectos psíquicos. Como hemos visto, el superyó producto de la religión no se contenta siquiera con la renuncia a realizar los actos prohibidos: basta que detecte el deseo (o el intento de satisfacerlo al menos en la fantasía) para atormentar al sujeto con un castigo implacable. Este resultado (en el que la renuncia instintual ya no tiene pleno efecto absolvente) pasa a ser inevitable en el instante en que se imponen prescripciones obligatorias respecto al contenido de los deseos y el modo como cada uno debe perseguir su propia felicidad. En efecto, si la "moral" va más allá de exigir que la búsqueda de la propia felicidad (por el camino que cada uno considere más oportuno) se ajuste, digamos, "a la forma de ley", es decir, que resulte compatible con el derecho de todos los demás a perseguir un fin semejante; si la "moral" va más allá e impone determinados contenidos como intrínsecamente repugnantes 0 deseables, el superyó castigará los "deseos repugnantes" incluso si se renuncia a realizarlos.

Este modo de proceder, ciertamente, le es ajeno a la razón. La valoración intrínseca de los contenidos (es decir, independiente de su compatibilidad o no con la forma de una posible legislación universal) es un asunto de interés para los catecismos, pero no un principio de la libertad (a la que sólo le puede preocupar el modo de hacer compatible la libertad de cada uno con la de todos los demás según reglas de validez general). Cabe decir que a la razón no le repugnan nada las "cochinadas" (Schweinerein); sólo le repugnan las injusticias y, en este sentido, ningún deseo le puede escandalizar. La

\footnotetext{
${ }^{44}$ Das Unbehagen in der Kultur, Gesammelte Werke, XIV, p. 443 [EI malestar en la cultura, Obras Completas, XXI, p. 84].

${ }^{45}$ Beantwortung der Frage: Was ist Aufklärung?, AA: VIII, 41.
} 
cuestión de la justicia no remite ciertamente al contenido ni al valor intrínseco de los deseos sino al derecho de los demás a realizar también los suyos propios en libertad. En este sentido, la preocupación de la razón (a diferencia de la de los clérigos) no versa sobre la validez esencial de los contenidos sino sobre las reglas de compatibilidad de unos contenidos con otros. Por lo tanto, no es competencia de la razón luchar, digamos, contra el contenido de los deseos (por escandalosos que resulten para la religión), sino, en todo caso, preguntarse por el margen de compatibilidad de los distintos deseos entre sí. Al igual que Freud, también Kant tiene claro que la razón y la libertad no tienen que combatir "contra carne y sangre (las inclinaciones naturales) sino contra príncipes y poderosos" 46 . Ciertamente, el problema para Kant no es nunca la presencia de deseos con los contenidos más variopintos sino, en todo caso, que se permita a los deseos determinar la voluntad generando máximas que de ningún modo puedan valer como leyes. El asunto, en todo caso, no remite a la validez intrínseca de los deseos en cuestión sino al problema de su compatibilidad, en caso de ser realizados, con los derechos y libertades de los demás. En este sentido, la razón, sin duda, pondrá el grito en el cielo, por ejemplo, ante el parricidio -y, quizá, ante el incesto (en la medida en que quizá sí disolviera las condiciones de toda sociedad humana posible)-, pero sólo ante el parricidio (o en su caso, el incesto) efectivos. Por el contrario, la razón tiene poco que objetar a cualquier deseo (incluido el parricidio y el incesto) que acepte quedar satisfecho sin generar efectos (o sea, que acepte quedar satisfecho, por decirlo así, "con meras palabras"). No es fácil ver de qué modo los deseos satisfechos en la imaginación o en el sueño pueden resultar en algún sentido formalmente incompatibles con la forma de ley. En efecto, la compatibilidad de unos deseos con otros satisfechos en la imaginación es realmente inagotable. Así, desde el punto de vista de la razón, el veredicto es inapelable: si se ha renunciado a producir efectos, que cese de inmediato el castigo. A diferencia de las religiones, que veneran ante todo a los santos, la razón celebra ante todo que los humanos, atravesados por una diversidad infinita de inclinaciones, se puedan comportar en este mundo con respeto por la forma de ley ${ }^{47}$. Por lo tanto, la razón permitirá estar "satisfecho consigo mismo" a quien logre desplazar a la "mera imaginación" deseos que serían incompatibles con la forma de ley en caso de realizarse. "¿Qué hace aquí el psicoanálisis sino corroborar el viejo dicho

\footnotetext{
${ }^{46}$ Die Religion innerhalb der Grenzen der bloßen Vernunft , AA: VI, 59.

${ }^{47}$ "El estado moral en el que puede estar siempre es la virtud, es decir, la intención moral en lucha, y no la santidad en supuesta posesión de una completa pureza en las intenciones de la voluntad" KpV, AA: V, 84. A este respecto, Kant no duda en advertir contra los peligros del "misticismo moral" (Cfr. KpV, AA: V, 86).
} 
de Platón, que los buenos son los que se conforman con soñar aquello que los otros, los malos, hacen realmente?" ${ }^{48}$.

\section{Minoría y mayoría de edad}

Dada la naturaleza humana y la existencia de la infancia, el mecanismo elemental de la neurosis resulta en cierto sentido insuperable. Sin embargo desde el punto de vista de la salud y la felicidad (y mucho menos desde el punto de vista de la razón y la libertad) no es en absoluto indiferente el nivel de presión que imponen en cada caso las exigencias y pretensiones de la cultura y el modo que tienen de imponerla. La máxima libertad no suprimiría el mecanismo elemental de la neurosis, pero sí podría reducir la presión sobre el sujeto a un mínimo más fácilmente gestionable sin producir patologías. En efecto, Freud demuestra cómo los mismos hechos infantiles producen efectos psíquicos muy distintos dependiendo de las normas y convenciones sociales a las que esté tratando de ajustarse el psiquismo en cuestión. En su Introducción al psicoanálisis, Freud explica esto recurriendo a la obra de Nestroy En el bajo y en el principal: la hija del portero (familia humilde que vive en el bajo) y la del propietario (familia de superior desarrollo "moral e intelectual" que vive en el principal), mantienen un contacto sexual infantil que determina sus vidas de un modo radicalmente diferente. La primera no ve alterado en absoluto su desarrollo por este hecho, pero en la segunda su psiquismo logra desplegar toda una estrategia de tortura que le arruina la vida. La causa de estos "dos destinos" es, ciertamente, el distinto nivel de presión que las normas y convenciones ejercen sobre ellas. "A la hija del portero, la práctica sexual le parecerá más tarde tan natural y tan sin reparos como en la infancia. La hija del propietario ha experimentado la influencia de la educación y aceptado sus exigencias" 49.

\footnotetext{
${ }^{48}$ Vorlesungen zur Einführung in die Psychoanalyse, Gesammelte Werke, XI, p. 147 [Introducción al psicoanálisis, Obras Completas, XV, p. 134].

${ }^{49}$ Vorlesungen zur Einführung in die Psychoanalyse, Gesammelte Werke, XI, p. 367 [Introducción al psicoanálisis, Obras Completas, XVI, 322]. El ejemplo de la homosexualidad le resulta a Freud especialmente ilustrativo. De hecho, llega a sostener que "en ningún neurórico faltan mociones homosexuales y que gran parte de los síntomas expresan esta inversión latente" Vorlesungen zur Einführung in die Psychoanalyse, Gesammelte Werke, XI, p. 318 』ntroducción al psicoanálisis, Obras Completas, XVI, p. 281]. En el marco de la teoría psicoanalítica, esta afirmación no es nada desconcertante. Si en la infancia el objeto está sin fijar de un modo centralizado y, por lo tanto, el deseo se dirige de un modo relativamente indeterminado hacia cualquier objeto que pudiera proporcionar placer, resulta elemental que, en su caso, la represión se ejercerá con efectos
} 
Para la teoría psicoanalítica, detrás de toda neurosis hay, por definición, algún deseo (sexual) reprimido. Ciertamente, el hecho de que una norma social genere un malestar (o una patología) individual no basta todavía para determinar si el problema está en la norma o en el individuo, es decir, no basta para decidir cuál de las dos cosas exige ser transformada. En efecto, podría ocurrir que un psiquismo determinado desencadenase un comportamiento patológico ante la simple prohibición, siquiera, de esos elementos mínimos sin los cuales es imposible la vida (civil) en común (elementos que hemos cifrado en el respeto a la libertad y la integridad ajenas). Por lo tanto, es evidente que la pauta y el criterio no la puede marcar, sin más, la "salud". A hora bien, desde cualquier planteamiento ilustrado, es también evidente que la pauta y el criterio tampoco nos la pueden proporcionar las propias reglas y convenciones sociales por el hecho de serlo. En efecto, podría ocurrir que cualquier clericalismo tratara de imponer con carácter general las regulaciones de su doctrina y, en ese sentido, generase una situación represiva intolerable.

A hora bien, estas situaciones represivas desproporcionadas reclaman, para Freud, una intervención tanto individual como social. Desde el punto de vita individual, la intervención terapéutica debe sacar a la luz los contenidos reprimidos para que, en la medida de lo posible, los gestione la conciencia y la razón (es decir, esa instancia a la que no le repugnan nada más que las injusticias) en vez del superyó (es decir, esa instancia en la que cualquier representante del clericalismo puede instalarse subrepticiamente a hablar con la solemnidad de la autonomía moral). Por su parte, la intervención "terapéutica" social propuesta por Freud pasa, de un modo enteramente análogo, por sacar a la luz las convenciones en las que se basa la represión y someterlas, en este caso, al escrutinio no de la razón individual sino, por decirlo así, de la razón pública. El primer modo de sacar a la luz, desde un punto de vista clínico, los contenidos de la represión es propiamente el psicoanálisis; el segundo (firmemente defendido por Freud e inseparable de todo su planteamiento) es, ciertamente, la Ilustración.

El compromiso implacable de Freud con el programa político de la Ilustración se pone de manifiesto en su crítica a la religión. En efecto, en El porvenir de una ilusión, que él describe como un "alegato en favor de fundamentar los preceptos culturales sobre la pura razón" 50 , define la religión como "la neurosis obsesiva humana"51 y, como no puede ser de otro modo,

\footnotetext{
${ }^{50}$ Die Zukunft einer Illusion, Gesammelte Werke, XIV, p. 365 [EI porvenir de una ilusión, Obras Completas, XXI, p. 41].

${ }^{51}$ Die Zukunft einer Illusion, Gesammelte Werke, XIV, p. 366 [EI porvenir de una ilusión, Obras Completas, XXI, p. 3].
} 
propone también a este respecto "sustituir los resultados de la represión por los del trabajo intelectual acorde a la razón" 52 .

El problema de la religión, ciertamente, es su empeño en tratar eternamente a los hombres y mujeres como menores de edad. La infancia biológica (esa "minoría de edad" de la que no hay más responsable que la Naturaleza), al carecer de herramientas racionales, no puede evitar recurrir a procedimientos afectivos para reprimir y dominar exigencias instintivas y, en esa medida, resulta inevitable el tránsito por un cierto proceso de "neurosis". Ciertamente, no está en nuestra mano eliminar la infancia. El hombre adquiere las normas (tanto las que se basan en un fundamento racional como las que no) al mismo tiempo que el lenguaje. Por lo tanto, resulta imposible que se trate de una adquisición racional y consciente por parte del sujeto. En efecto,

Acerca de los niños, sabemos que no pueden recorrer bien su camino de desarrollo hacia la cultura sin pasar por una fase de neurosis, ora más nítida ora menos. Esto se debe a que el niño no puede sofocar, mediante un trabajo intelectual acorde a la razón, considerable número de sus exigencias pulsionales inválidas para su vida posterior, sino que debe domeñarlas mediante actos de represión tras los cuales se encuentra, por regla general, un motivo de angustia. ${ }^{53}$

En este sentido, resulta constitutivamente insuperable la incapacidad (en la infancia) de someter racionalmente los impulsos proscritos y, por lo tanto, resulta inevitable el recurso a la represión afectiva (origen de la neurosis). En todo caso, según Freud, si la cultura no impusiera, por esta vía represiva, unas exigencias desproporcionadas, estas "neurosis" infantiles se disolverían en el transcurso del crecimiento (o, en su caso, podrían desactivarse en la edad adulta a través de la intervención psicoanalítica).

Sin embargo, la cuestión se complica por la persistencia de algo a lo que cabría quizá llamar "minoría de edad estructural” (o social) y que funciona de un modo enteramente análogo al de la neurosis individual.

De manera en un todo parecida, cabría suponer que la humanidad en su conjunto, en el curso de su secular desarrollo, cayó en estados análogos a las neurosis, y sin duda por las mismas razones: porque en las épocas de su ignorancia y su endeblez intelectual, las renuncias de lo pulsional

\footnotetext{
${ }^{52}$ Die Zukunft einer Illusion, Gesammelte Werke, XIV, p. 368 [EI porvenir de una ilusión, Obras Completas, XXI, p. 44].

${ }^{53}$ Die Zukunft einer Illusion, Gesammelte Werke, XIV, p. 366 [EI porvenir de una ilusión, Obras Completas, XXI, p. 42].
} 
indispensables para la convivencia humana sólo podían obtenerse a través de una fuerzas puramente afectivas. Y luego quedaron por largo tiempo adheridas a la cultura las sedimentaciones de esos procesos, parecidos a una represión, acaecidos en la prehistoria. ${ }^{54}$

El paralelismo entre la dimensión individual y social es, para Freud, casi absoluto: por lo mismo por lo que se denomina "infancia" a carecer de herramientas con las que controlar, a través de la razón, las exigencias instintivas, sólo cabría denominar "mayores de edad" a esas sociedades en las que bastaran los escuetos principios de la razón para regular las relaciones de los hombres entre sí, es decir, esas sociedades en las que toda prohibición, restricción o coacción se ejerciese siempre de un modo acorde al principio de libertad antes señalado (según el cual "nadie me puede obligar a ser feliz a su modo").

Esta posibilidad de edificar una sociedad (y regular las relaciones de los hombres entre sí) sin necesidad de prohibir coactivamente nada que no atente contra la libertad y la integridad de los demás es a lo que Kant y toda la tradición ilustrada ha denominado "estado civil republicano" o "estado de derecho", cuyo opuesto directo es, como hemos dicho, el gobierno paternalista (imperium paternale) $)^{55}$.

A hora bien, del mismo modo que el correlato necesario del estado de derecho son los ciudadanos libres (a los que se supone al menos un mínimo de virtud republicana), el correlato necesario de un gobierno paternalista son los súbditos infantiles (a los que se supone al menos un mínimo de obediencia y consentimiento). Este carácter infantil remite, tanto en Freud como en Kant, a la incapacidad de "servirse del propio entendimiento sin la guía de otro" y a la necesidad de buscar un referente exterior que establezca "qué debo hacer". Lo que aporta Freud es una extraordinaria claridad al análisis de los mecanismos por los que esta, digamos, "minoría de edad estructural" garantiza su reproducción. En determinadas condiciones educativas (a las que, en general, cabría denominar "atmósfera religiosa"),

\footnotetext{
${ }^{54}$ Die Zukunft einer Illusion, Gesammelte Werke, XIV, p. 366-367 $\mathbb{E} \mid$ porvenir de una ilusión, Obras Completas, XXI, p. 43]. Del mismo modo, en EI malestar en la cultura, Freud se pregunta: “ ¿no se está justificado en diagnosticar que muchas culturas - o épocas culturales- y aun posiblemente la humanidad toda, han devenido 'neuróticas' bajo el influjo de las aspiraciones culturales?" Das Unbehagen in der Kultur, Gesammelte Werke, XIV, p. 504 [EI malestar en la cultura, Obras Completas, XXI, p. 139].

${ }^{55}$ En efecto, para Kant, el derecho no es nada más que "el conjunto de condiciones bajo las cuales el arbitrio de uno puede conciliarse con el arbitrio del otro según una ley universal de la libertad", Die Metaphysik der Sitten, AA: VI, 230.
} 
cuanto más intentan los sujetos adultos hablar con autonomía o escuchar a su propia conciencia, más le dan la palabra no a la voz de su razón sino a la voz de su padre (o, lo que es lo mismo, a la voz de ese dios estatutario que prescribe y prohíbe también con mandatos de la conciencia). A hora bien, esos adultos que se comportan como niños ante las amenazas Dios, es decir, esos adultos cuya moral sí necesita de la religión para mantenerse en pie, son, evidentemente, los padres que educan a los niños de una "sociedad menor de edad". A partir de aquí, el bucle de la reproducción (como en cualquier estructura) logra cerrarse: la minoría de edad "social" o "estructural" es, a la vez, causa y efecto de la minoría de edad de sus adultos y, en esta reproducción, está garantizado, a través de la educación diseñada por los padres, que el desarrollo de los niños representa un tránsito de la minoría de edad biológica a la minoría de edad moral (es decir, el tránsito de un estado en el que, en ausencia de razón, no hay autoridad más alta que el padre a un estado en el que no hay autoridad más alta que el dios de nuestros padres, interpelándonos, eso sí, a través de nuestra propia conciencia).

En este sentido, cabe advertir que la mayoría de edad (como concepto político y moral) no se alcanza automáticamente, ni mucho menos, en la edad adulta, pero ni siquiera en una edad adulta firmemente dispuesta a proceder de un modo autónomo, sino sólo en una edad adulta con suficiente valor para servirse de su propio entendimiento en una sociedad mayor de edad.

El propio Kant sospecha ya con nitidez que no cabe lograr la salida del hombre de su autoculpable minoría de edad a una escala individual sino que, por el contrario, es una tarea que sólo está al alcance de una intervención colectiva (a través del uso público, común y compartido, de la razón libre). En efecto, Kant sostiene que

son pocos los que, por esfuerzo del propio espíritu, han conseguido salir de esa minoría de edad y proseguir, sin embargo, con paso seguro.

Pero, en cambio, es posible que el público se ilustre a sí mismo, algo que es casi inevitable si se le deja en libertad ${ }^{56}$.

\section{La organización republicana como condición de la autonomía moral}

Pero ¿cómo se deja a la razón pública en libertad? El problema aquí ya no es sólo garantizar la libertad de hacer siempre y en todo lugar uso público de la propia palabra, sino también generar unas condiciones en las que "yo" pueda significar en los adultos algo distinto a la cristalización interior de las

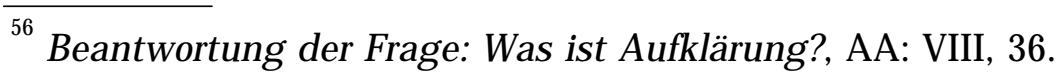


costumbres de los ancestros, es decir, pueda significar la capacidad efectiva de hacer uso de la propia razón. Y, para ello, es imprescindible desactivar el bucle que se basa en el diseño exclusivo de la educación por parte de los padres.

A hora bien, para lograr este objetivo no basta en absoluto con desactivar las instituciones exteriores de la cultura. Más bien al contrario, este es un asunto que exige la máxima cautela. La razón no se puede en absoluto desentender de las funciones efectivas que desempeña la cultura. Si desmantela sus instituciones, no suprime el problema sino que, simplemente, obliga a los sujetos a gestionarlo a una escala individual y, de este modo, los condena a desarrollar todo tipo de psicopatologías. En efecto, el colmo en una sociedad menor de edad sería retirar las instituciones sociales que ayudan al sujeto a cumplir las restricciones abusivas que impone la religión y obligarle a gestionar esa represión por sus propios (y limitados) medios ${ }^{57}$. Se debe recordar que, dado el peculiar mecanismo con el que opera el psiquismo, liquidando las instituciones sociales a las que cabría identificar como responsables de la opresión no se obtiene como resultado ninguna liberación sino, simplemente, la destrucción de las herramientas colectivas que ayudan a gestionar la represión de un modo viable. En ausencia de ellas, el superyó individual no sería más indulgente en sus pretensiones. Simplemente, estaría más aislado en su ejercicio y, por lo tanto, se canalizarían como patologías individuales todos los elementos que la cultura era capaz de gestionar con indiscutible eficacia.

En este sentido, el proyecto político de la Ilustración debe evitar que esas pretensiones abusivas penetren como constitutivo del sujeto en la infancia. Para ello, no puede eludir como su principal obligación la elaboración de un programa pedagógico basado en los principios de la razón y la libertad. En efecto, resulta crucial la organización de instituciones que preparen a los niños para el ejercicio de su libertad, es decir, que orienten la educación no hacia la inserción en una comunidad religiosa sino en una comunidad civil. Para ello, la educación debe abrir desde el principio un amplio margen para la búsqueda y satisfacción de los propios fines, marcando el límite en la compatibilidad con la libertad de los otros. A sí pues, para Kant es evidente que "el niño tiene que sentir siempre su libertad, de manera tal que no obstaculice la libertad de otros" 58 . Y esta condición debe plantearse

\footnotetext{
${ }^{57}$ Para un tratamiento más amplio de esta cuestión, Cf. Fernández Liria, Geometría y tragedia, Hondarribia: Hiru, 2001.

${ }^{58}$ Päd., AA: IX 464. Para un extraordinario análisis de la cuestión de la pedagogía en Kant, Cf. Nuria Sánchez Madrid, Egoísmo, disciplina y libertad regulada: observaciones conjeturales sobre el primer despliegue de la libertad en la naturaleza del hombre (vd. bibliografía).
} 
precisamente como garantía de la propia libertad (es decir, como único modo de evitar que la vida en común se convierta en una selva en la que únicamente goza de libertad el más fuerte, y sólo de un modo provisional).

En esta dirección, Kant propone tres pasos necesarios que debe seguir el programa pedagógico para una inserción libre de los niños en la comunidad:

a) debe dejarse en libertad al niño en todos los contextos, exceptuando las situaciones en que tiene cerca objetos con los que puede lastimarse, y siempre que su libertad no choque con la de los otros (si grita o se divierte de manera escandalosa molestará al prójimo).

b) debe indicársele que sólo puede alcanzar sus propios fines si permite que los demás también los alcancen, lo cual se logra negándole todo divertimento mientras no haga lo que se le dice, de suerte que deponga voluntariamente sus accesos de rabia una vez comprobada su ineficacia.

c) hay que demostrarle que la coacción a la que se le somete es el medio para conducirle al uso cabal de su propia libertad, de manera que habrá que cultivar el sometimiento hasta que pueda ser libre de verdad, liberándose así del cuidado ajeno, lo que sólo ocurre al final del proceso..$^{59}$

El objetivo, en cualquier caso, debe ser desactivar la atmósfera religiosa en lo relativo a la educación y, por lo tanto, garantizar un desarrollo libre de la vida del sujeto. Así pues, por lo que respecta a la infancia, la Ilustración no puede eludir una lucha sin cuartel contra esa instancia "que viene a perturbar este libre juego de elección y adaptación, al imponer a todos por igual su camino único para alcanzar la felicidad y evitar el sufrimiento". Con este objetivo, la primera máxima irrenunciable del programa pedagógico republicano, en lo relativo a los representantes del clericalismo, debe ser "impedir que los niños se acerquen a ellos" (para evitar que resulten víctimas inermes de la represión sexual desmedida).

Sólo después de Freud es posible calibrar la verdadera dimensión de la importancia que corresponde a estas instituciones educativas republicanas. En efecto, sólo después de Freud sabemos hasta qué punto un programa pedagógico ilustrado y republicano constituye una condición necesaria tanto de la libertad civil como, incluso, de la autonomía moral. En ausencia de una educación libre, diseñada y ejercida desde la razón pública, el resultado inevitable será que la voz del padre (o de su dios), clamando desde el interior de la conciencia, suplante y ahogue la voz de la razón. No obstante, "la voz

${ }^{59}$ Päd., AA: IX 454. 
del intelecto es leve, mas no descansa hasta ser escuchada. $Y$ al final lo consigue, tras incontables, repetidos rechazos". ${ }^{60}$

ALEGRE ZAHONERO, Luis. Civil freedom as a condition of possibility of moral autonomy. On Freud's contribution to Kant's practical approach. Trans/Form/Ação, (São Paulo); v.33(1), 2010, p.155-182.

\begin{abstract}
It is usual to read Freud as a critic of Enlightenment, specialy of the kantian way of thinking moral autonomy. However, all Freud did was discover in human nature a great difficulty that enlightenes philosophy just cannot ignore. This difficulty, which directly affects conceptions about the age of majority, refers to certain heteronomy mechanisms which main characteristic is the skill to supplant the voice of moral autonomy: exactly the same as moral law in Kant, super-ego -once internalized during childhood and once culture imperatives have been assumed as one's own (regardless of their rationality)- orders without promising anythig (external) or threatening anything (external). So, the political project of enthroning reason as the top authorithy (project shared by both, Kant and $F$ reud) depends on the existence of a republican organization that guarantees a pedagogical program for which "age of majoriry" does not mean any more naming "ego" to ancient customs internalized but the capacity of being ruled by one's own reason (which becomes, once psychoanalysis developed, not only a moral imperative but also a therapeutic imperative).
\end{abstract}

KE YWORDS: Freud. Kant. Age of majority. M inority. Enlightenment.

\title{
Bibliografía
}

\section{Obras fuente}

FREUD, S. Totem und Tabu. Hamburg: S. Fischer Verlag, 1973. (Gesammelte Werke, IX). Traducción: Tótem y tabú. Traducción J osé Luis Etcheverry. Buenos Aires: A morrortu, 1991. (Obras Completas, XIII).

Vorlesungen zur Einführung in die Psychoanalyse. Hamburg: S. Fischer Verlag, 1973. (Gesammelte Werke, XI). Traducción: Conferencias de introducción al psicoanálisis. Traducción J osé Luis Etcheverry. Buenos Aires: Amorrortu, 1994. (Partes I y II: Obras Completas, XV; Parte III: Obras Completas, XVI).

. Die Zukunft einer Illusion. Hamburg: S. Fischer Verlag, 1972. (Gesammelte Werke, XIV, p. 321-380). Traducción: El porvenir de una llusión. Traducción J osé Luis Etcheverry. Buenos Aires: Amorrortu, 1998. (Obras Completas, XXI).

\footnotetext{
${ }^{60}$ Die Zukunft einer Illusion, Gesammelte Werke, XIV, p. 377 [EI porvenir de una ilusión, Obras Completas, XXI, p. 52.] J osé Luis Villacañas plantea una pregunta fundamental al hilo de la relación entre Blumenberg, Kant y Freud: "lo que sabemos del ser humano, ¿nos permite esperar su ilustración" ("Dificultades con la Ilustración", A raucaria: Revista Iberoamericana de filosofía, política y humanidades, p. 27). Como hemos tratado de defender, la respuesta freudiana es afirmativa: quizá nada nos permite garantizar que esa ilustración vaya a tener lugar necesariamente, pero sí cabe esperar que sea posible.
} 
FREUD, S. Das Unbehagen in der Kultur. Hamburg: S. Fischer Verlag, 1972. (Gesammelte Werke, XIV, p. 419-506). Traducción: EI malestar en la cultura. Traducción J osé Luis Etcheverry. Buenos Aires: Amorrortu, 1998. (Obras Completas, XXI).

. Neue Folge der Vorlesungen zur Einführung in die Psychoanalyse. Hamburg: S. Fischer Verlag, 1973. (Gesammelte Werke, XV). Traducción: Nuevas conferencias de introducción al psicoanálisis. Traducción J osé Luis Etcheverry. Buenos Aires: A morrortu, 1991. (Obras Completas, XXII).

Der Mann Moses und die monotheistische Religion. Hamburg: S. Fischer Verlag, 1972. (Gesammelte Werke, XVI, p. 101-246). Traducción: Moises y la religión monoteísta. Traducción J osé Luis Etcheverry. Buenos Aires: A morrortu, 1993. (Obras Completas, XXIII).

KANT, I. Kritik der praktischen Vernunft [AA. V]. In: Gesammelte Schriften. Ed. A kademie der Wissenschaften. Berlin: De Gruyter, $1900 \mathrm{ff}$. Traducción: Crítica de la razón práctica. Traducción E. Miñana y Villagrasa y Manuel García Morente. Salamanca: Ediciones Sígueme, 1998.

. Die Religion innerhalb der Grenzen der bloßen Vernunft [AA. VI]. In: . Gesammelte Schriften. Ed. Akademie der Wissenschaften. Berlin: De Gruyter, 1900ff. Traducción: La religión dentro de los límites de la mera Razón. Traducción Felipe Martínez Marzoa. Madrid: Alianza, 1995.

Die M etaphysik der Sitten [AA. VI]. In: . Gesammelte Schriften. Ed. A kademie der Wissenschaften. Berlin: De Gruyter, 1900ff. Traducción: Metafísica de las costumbres. Traducción Adela Cortina Orts y J esús Conill Sancho. Madrid: Tecnos, 2002.

Beantwortung der Frage: Was ist Aufklärung? [AA. VIII]. In: Gesammelte Schriften. Ed. A kademie der Wissenschaften. Berlin: De Gruyter, 1900ff. Traducción: "Respuesta a la pregunta: ¿qué es Ilustración?", en ¿Qué es Ilustración. Traducción Agapito M aestre y J osé Romagosa. Madrid: Tecnos, 1993.

Über den Gemeinspruch: Das mag in der Theorie richtig sein, taugt aber nicht für die Praxis [AA. VIII]. In: Gesammelte Schriften. Ed. A kademie der Wissenschaften. Berlin: De Gruyter, 1900ff. Traducción: “En torno al tópico: 'tal vez eso sea correcto en teoría, pero no sirve para la práctica'", en Teoría y práctica. Traducción Francisco Pérez López y Roberto Rodríguez A ramayo: Madrid: Tecnos, 1993.

. Von einem neuerdings erhobenen vornehmen Ton in der Philosophie [AA. VIII]. In: _-. Gesammelte Schriften. Ed. Akademie der Wissenschaften.Berlin: De Gruyter, 1900ff. Traducción: “De un tono distinguido, recientemente ensalzado en Filosofía", en En defensa de la llustración. Traducción J avier Alcoriza y A ntonio Lastra. Barcelona: Alba Editorial, 1999. 
FREUD, S. Pädagogik (AA . IX). In: . Gesammelte Schriften. Ed. A kademie der Wissenschaften. Berlin: De Gruyter, $1900 \mathrm{ff}$.

\section{Otras Referencias}

CALLEJ O HERNANZ, M. J . Ley, deseo y libertad. Notas sobre Lacan y la Crítica de la razón práctica. Documento de trabajo del grupo M etafísica, Crítica y Política (UCM -930566), en el marco del Proyecto de Investigación "Naturaleza Humana y Comunidad" (HUM 2006-04909).

FERNÁNDEZ LIRIA, C. Geometría y tragedia. Hondarribia: Hiru, 2001.

FUENTES ORTEGA, J . B. De Kant a Freud: la formación del sujeto modernista en el seno de las crisis románticas del pensamiento kantiano. Documento de trabajo del grupo Metafísica, Crítica y Política (UCM-930566), realizado en el marco del Proyecto de Investigación "Naturaleza Humana y Comunidad" (HUM 2006-04909) dirigido por J uan Manuel Navarro Cordón. 2009.

FULGENCIO, L. Kant e as especulações metapsicológicas em Freud. Kant ePrints, v. 2, n. 9, p. 1-31, 2003.

LOPA RIC, Z. De Kant a Freud: um roteiro. Natureza Humana, v. 5, n. 1, p. 231245, 2003.

SÁNCHEZ MADRID, N. Egoísmo, disciplina y libertad regulada: observaciones conjeturales sobre el primer despliegue de la libertad en la naturaleza del hombre. Ponencia presentada en el seminario de investigación "Razón, libertad y educación. Una discusión a partir de Kant", organizado en el marco del Proyecto de Investigación UCM "Lenguaje, Pedagogía y Derecho. Un problema de la Antropología moral de Kant" (CCG08-UCM/HUM-4166) (Disponible próximamente en e-prints UCM).

SANZ FERRAM OLA, R. El imperativo categórico de Kant en Freud. Fundamentos en Humanidades, n. 3, p. 167-187, 2001.

VILLACAÑAS BERLANGA, J. L. Dificultades con Ia Ilustración. Araucaria: Revista Iberoamericana de Filosofía, Política y Humanidades, n. 21, p. 27-43, 2009. 
